\title{
The CLIP-Substituted Invariant Chain Efficiently Targets an Antigenic Peptide to HLA Class II Pathway in L Cells
}

\author{
Shinji Fujii, Satoru Senju, Yu-Zhen Chen, \\ Masayuki Ando, Sho Matsushita, and \\ Yasuharu Nishimura
}

\begin{abstract}
The presentation of antigenic peptides by major histocompatibility complex (MHC) class II to $\mathrm{CD}^{+}{ }^{+} \mathrm{T}$ cells is crucial to initiate immune responses. We developed a new system for delivery of an antigenic peptide to the MHC class II pathway, using the invariant chain (Ii). We designed a mutated human p33-form Ii, CLIP-substituted Ii, in which streptococcal M12p55-68 (RDLEQAYNELSGEA) was substituted for CLIP (class II associated invariant chain peptide). We examined the peptide presenting function of this construct, in comparison with the previously reported C-terminal fused $\mathrm{Ii}$, in which a cathepsin cleavage site and M12p54-68 was ligated to the C-terminus of Ii. Mouse L cell transfectants expressing either of these two mutated Ii along with HLA-DR 4 could process and present M12p55-68 to the
\end{abstract}

peptide specific and DR4-restricted $\mathrm{CD} 4^{+} \mathrm{T}$ cell clone. CLIP-substituted Ii was much more efficient in antigen presentation than was the C-terminal fused Ii. Similar to the wild-type Ii, the CLIP-substituted Ii was associated intracellularly with DR 4 molecules. These results indicate that the peptide substituted for CLIP of Ii p33 bound to the groove of DR molecules in the same manner as CLIP and it was preferentially presented to the $\mathrm{CD}^{+}{ }^{+} \mathrm{T}$ cell clone in the absence of HLA-DM molecules. This system may prove useful for immunotherapy with DNA vaccines or for construction of an antigen presenting cell library with diverse peptides. Human Immunology 59, 607-614 (1998). (C) American Society for Histocompatibility and Immunogenetics, 1998. Published by Elsevier Science Inc.

\begin{tabular}{|c|c|c|c|}
\hline \\
\hline \multicolumn{4}{|c|}{$\begin{array}{l}\text { ABBREVIATIONS } \\
\text { APC antigen presenting cell }\end{array}$} \\
\hline CLIP & class II-associated invariant chain peptide & $\mathrm{mAb}$ & monoclonal antibody \\
\hline HLA & human leukocyte antigen & $\mathrm{MHC}$ & major histocompatibility complex \\
\hline
\end{tabular}

\section{INTRODUCTION}

The recognition of antigenic peptides in the context of major histocompatibility complex (MHC) class II by $\mathrm{CD}^{+} \mathrm{T}$ cells is crucial to initiate immune responses. Establishment of a system for efficient delivery of anti-

From the Division of Immunogenetics, Department of Neuroscience and Immunology (S.F., S.S., Y-Z.C., S.M., Y.N.), Kumamoto University Graduate School of Medical Sciences, Kumamoto University School of Medicine, Kumamoto 860-0811, Japan, and the First Department of Internal Medicine (S.F., M.A.), Kumamoto University School of Medicine, Kumamoto 860-0811, Japan.

Address reprint requests to: Yasubaru Nishimura, M.D., Division of Immunogenetics, Department of Neuroscience and Immunology, Kumamoto University Graduate School of Medical Sciences, Honjo 2-2-1, Kumamoto 860-0811, Japan. Tel: +81-96-373-5310; Fax: +81-96-373-5314; E-Mail:mxnishim@gpo.kumamoto-u.ac.jp.

Received April 27, 1998; accepted May 22, 1998. genic peptides to the MHC class II pathway is an approach which will enhance immune responses of $\mathrm{CD}^{+}{ }^{+} \mathrm{T}$ cells and should be useful for development of a potent vaccine. For this purpose, a system is needed to deliver a given specific antigen onto MHC class II molecules and a strategy of introducing gene encoding antigenic peptide to antigen presenting cells (APC) is useful in many respects. However, except for membrane or secretory proteins, many proteins endogenously synthesized by gene introduction as well as viral infection are degraded into peptide fragments by proteasomes in cytoplasm, which are transported into the lumen of the endoplasmic reticulum by the transpoter associated with antigen processing (TAP). The peptides then bind to MHC class I molecules and are presented to $\mathrm{CD} 8^{+} \mathrm{T}$ cell $[1]$. The use 
of antigenic molecules with targeting signals to endosomes is a pertinent strategy for antigen presentation to $\mathrm{CD}^{+}{ }^{+} \mathrm{T}$ cell via class II pathway by gene introduction. Several systems have been reported to use various molecules with targeting signals, including cathepsin D [2], lysosomal-associated membrane protein LAMP-1 [3], and the invariant chain [4-6].

The invariant chain is type II integral membrane glycoproteins. The p33 and p35 forms of Ii, both encoded by eight exons, differ by $16 \mathrm{~N}$-terminal residues as a result of alternative translational initiation [7]. The p41 Ii, encoded by nine exons, is generated by alternative splicing of a common pre-mRNA. Theoretically, an additional p43 form could be expected [8], but is not yet unequivocally detected in immunoprecipitates from human cells. Among Ii isoforms, the p33 form is predominant in the human Ii chain. Ii has two known important functions with regard to antigen presentation by MHC class II molecules: First, Ii associates with newly synthesized MHC class II $\alpha$ and $\beta$ dimers in the endoplasmic reticulum to form a complex and directly prevents peptides in the endoplasmic reticulum from binding to MHC class II molecules, using a domain termed class II-associated Ii peptide (CLIP) that occupies the peptidebinding groove of class II $\alpha \beta$ dimers $[9-11]$. This $\alpha \beta$-Ii complex is composed of one Ii trimer and three $\alpha \beta$ dimers [12] and transported via the Golgi apparatus to the endosomal pathway by the targeting signal, dileucine motif, in the cytoplasmic domain of Ii $[13,14]$. In this pathway, Ii is proteolytically degraded and the $\alpha \beta$-CLIP complex is generated. In specialized endocytic organelles enriched in MHC class II molecules and designated MHC class II compartments (MIIC) $[15,16]$ or class II vesicles (CIIV) [17], HLA-DM catalyzes the dissociation of CLIP from class II $\alpha \beta$ dimer and binding of other peptides $[18,19]$.

Thus Ii contains not only the targeting signal to the endosomal pathway but also CLIP bound in the peptidebinding groove of MHC class II molecules. We reasoned that a CLIP-substituted Ii vector, encoding the Ii chain containing an antigenic peptide replacing CLIP, might efficiently load antigenic peptides on MHC class II molecules. We tested this thesis in comparison with the C-terminal fused Ii [5] and found that CLIP-substituted Ii is effective in targeting peptides to MHC class IImediated antigen presentation pathway.

\section{MATERIALS AND METHODS}

\section{Cells and Antibodies}

The $\mathrm{CD}^{+}{ }^{+} \alpha \beta$ T cell clone YN5-32 was established from a donor heterozygous for HLA-DRB1*0401 and HLADRB $1 * 0406$, by stimulating peripheral blood mononuclear cells with the peptide M12p54-68 (NRDLEQAY
NELSGEA), which corresponds to amino acid residues 54 to 68 of the $\mathrm{M}$ protein derived from a group A $\beta$ hemolytic strain 12 streptococcus, as described elsewhere [20]. The L cell transfectant LDR4 (B18D7) expressing the DRA gene plus DRB1*0406 gene [21] was distributed in the 11th International Histocompatibility Workshop [22]. For flow cytometry, the Fluorescein Isothiocyanate (FITC) conjugated mouse anti-human invariant chain $\mathrm{mAb} \mathrm{M}$ B741 and anti-HLA-DR mAb L243 were purchased from PharMingen (San Diego, CA) and Becton Dickinson Immunocytometry Systems (San Jose, CA), respectively. The mouse anti-human CLIP mAb CerCLIP.1 [23] was kindly provided by P. Cresswell (Yale University). FITC labeled goat anti mouse $\operatorname{IgG}+\operatorname{IgM}$ was purchased from BIOSOURCE (Camarillo, CA).

\section{Plasmid DNA Constructs}

The cDNA encoding human p33 Ii (American Type Culture Collection, Rockville, MD) was subcloned into the expression vector $\mathrm{pYN}$ containing the $\mathrm{SR} \alpha$ promoter [24]. The vector $\mathrm{pCI}$ was prepared, in which the DNA sequence encoding the CLIP region $\left({ }^{89}\right.$ SKMRMATPL LMQALPM $^{104}$ ) of Ii can be replaced by various sequences encoding antigenic peptides. The PinA I and Sac I sites were newly created by site-directed mutagenesis with mutagenic primers 5'-GCC TCC CAA $\underline{\text { ACC }}$ GGT GAG CAA GAT G-3' and 5'-CCA TGG GAG CIC TGC CCC AGG-3', using Transformer ${ }^{\mathrm{TM}}$ Site-Directed Mutagenesis Kits (CLONTECH, Palo Alto, CA). To generate pCIM, encoding mutant Ii containing M12p55-68 replacing CLIP, oligonucleotides 5'-CCG GTG CGT GAC TTG GAA CAA GCG TAT AAT GAG CTT AGC GGA GAA GCA CCC ATG GGA GCT- $3^{\prime}$ and 5'-CCC ATG GGT GCT TCT CCG CTA AGC TCA TTA TAC GCT TGT TCC AAG TCA CGC $A-3^{\prime}$ were annealed and subsequently ligated to pCI digested with the PinA I and Sac I. The C-terminal fused Ii vector pFIM, as reported [5], was constructed by inserting two dsDNA fragments in tandem after annealing the oligonucleotides 5'-GAT TTC TTA GAA GAA AAA AAA TCC GGA AAT CGT GAT-3' and 5'-CTA GAT CAC GAT TTC CGG ATT TTT TTT CTT CTA AGA AAT CC-3', 5'-CTA GAA CAA GCT TAT AAT GAG CTT AGC GGA GAA GCA TGA GAT-3' and 5'-CTA GAT CTC ATG CTT CTC CGC TAA GCT CAT TAT AAG CTT GTT- $3^{\prime}$ at the Xcm I site. These dsDNA fragments encode the cathepsin D cleavage site (FLEEKKSG) followed by M12p54-68 (NRDLEQAY NELSGEA) at the C-terminus of Ii.

\section{Flow Cytometry}

Cell surface staining for flow cytometric analysis was carried out as described elsewhere [20]. For intracellular staining, The cells were fixed in Dulbecco's Modified 
Eagle Medium (Gibco, Grand Island, NY) containing $1 \%$ paraformaldehyde (Wako, Osaka, Japan) for $10 \mathrm{~min}$ at room temperature and permeabilized by twice washing with Dulbecco's Modified Eagle Medium containing $0.1 \%$ saponin (SIGMA, St. Louis, MO) and $1 \%$ fetal calf serum. After incubation for $30 \mathrm{~min}$ at room temperature with the indicated $\mathrm{mAb}$ in the presence of $0.1 \%$ saponin and two additional washes, the cells were analyzed on FACScan (Becton Dickinson, Mountain View, CA).

\section{Metabolic Labeling and Immunoprecipitation}

Cells $\left(1 \times 10^{6}\right)$ grown in 6 well plate (Falcon, Becton Dickinson, Lincoln Park, NJ) were starved for $30 \mathrm{~min}$ in methionine- and cysteine-free Dulbecco's Modified Eagle Medium (Gibco) containing 2\% fetal calf serum. $\left.{ }^{35} \mathrm{~S}\right]$ methionine/cysteine (ICN, Irvine, CA) was added to a final concentration of $3.7 \mathrm{MBq} / \mathrm{ml}$, and the cells were incubated at $37^{\circ} \mathrm{C}$ for $2 \mathrm{~h}$. After removal of the medium the cells were washed twice with phosphate buffered saline then harvested from the wells by adding $1 \mathrm{ml}$ ice-cold lysis-buffer [phosphate buffered saline containing 1\% Nonidet P-40, 1 mM PMSF (SIGMA), and 1 $\mu \mathrm{g} / \mathrm{ml}$ pepstatin (SIGMA)]. After rocking the lysate at $4^{\circ} \mathrm{C}$ for $10 \mathrm{~min}$ the samples were centrifuged for $10 \mathrm{~min}$ at $15,000 \mathrm{~g}$ at $4^{\circ} \mathrm{C}$ to remove nuclei. For immunoprecipitation, $60 \mu \mathrm{l}$ of a slurry of protein A-Sepharose beads preabsorbed with HU-4 was added to the lysates followed by gentle rotation for $1 \mathrm{~h}$ at $4^{\circ} \mathrm{C}$. The beads were washed four times with phosphate buffered saline containing $1 \%$ Nonidet P-40 and twice with phosphate buffered saline. The beads were then boiled in sample buffer for $4 \mathrm{~min}$ and supernatants were applied to SDSPAGE. After electrophoresis, the gel was fixed, vacuum dried, and analyzed using radioautography or a fluorescence imaging system (FLA2000, Fuji Film, Tokyo, Japan).

\section{T Cell Proliferation Assay}

Antigen-specific proliferation of the $T$ cell clone was investigated, as described [25]. $\mathrm{L}$ cell transfectants were used as APC, and peptide pulse of $\mathrm{L}$ cell transfectants was done as follows: L cell transfectants were treated with $20 \mu \mathrm{g} / \mathrm{ml}$ of mitomycin C (SIGMA) as described [26], and 3.5 $\times 10^{4}$ cells/well were incubated with Dulbecco's Modified Eagle Medium supplemented with $10 \%$ fetal calf serum and the indicated peptide in a 96-well plate for $16 \mathrm{~h}$, followed by two washings with RPMI 1640 . The $\mathrm{T}$ cell clone $(3 \times$ $10^{4}$ ) was cultured with APC for $72 \mathrm{~h}$ then was pulsed with $1 \mu \mathrm{Ci} /$ well of $\left[{ }^{3} \mathrm{H}\right]$ thymidine for the last $16 \mathrm{~h}$. The cells were then harvested and the incorporated radioactivity was measured by liquid scintillation counting.
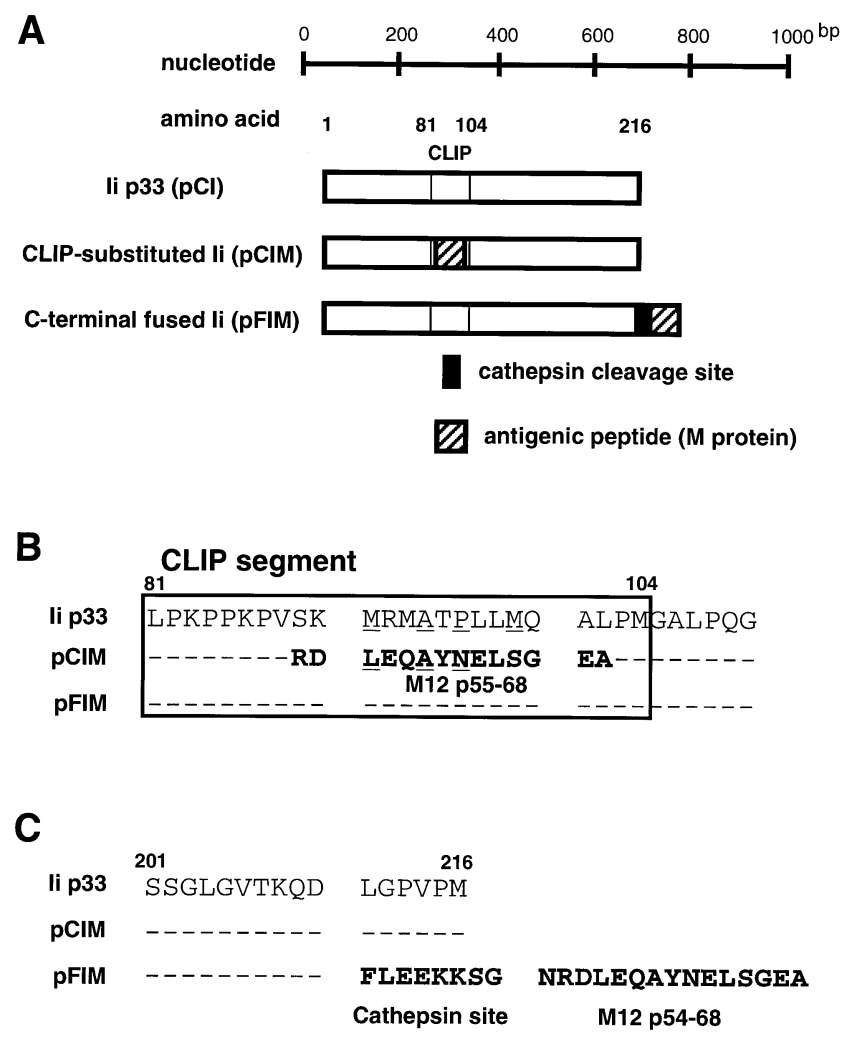

FIGURE 1 Diagram and amino acid sequences of constructs for Ii p33 form (pCI), CLIP-substituted Ii (pCIM) and C-terminal fused Ii (pFIM). (A) Diagram of the invariant chain, cathepsin $\mathrm{D}$ cleavage site, and the antigenic peptide derived from streptococcal $\mathrm{M}$ protein is indicated by white, black, and hatched bar, respectively. Amino acid sequences of CLIP-substituted Ii (B) and C-terminal fused Ii (C). The antigenic peptide substituted for CLIP, and the cathepsin D cleavage site and antigenic peptide added to the C-terminus of $\mathrm{Ii}$ are indicated by bold letters. The class II-associated invariant chain peptide (CLIP) is boxed. The HLA-DR anchor residues of CLIP and putative ones for the antigenic peptide M12p55-68 are underlined in panel B.

\section{RESULTS}

\section{Generation of Mutated Ii Genes}

As shown in Fig. 1, the CLIP-substituted Ii vector (pCIM) was created by inserting the sequence encoding the streptococcal M protein-derived peptide M12p55-68 (RDLEQAYNELSGEA), the epitope of HLA-DR4 (DRA+ DRB1*0406) restricted $\mathrm{CD}^{+}{ }^{+} \mathrm{T}$ cell clone $\mathrm{YN} 5-32$, into the CLIP region of pCI. The putative MHC first anchor residue for this epitope was assigned to be L57 [20]. On the other hand, CLIP was shown by X-ray crystal structure analysis to be bound to HLA-DR 3 molecules in a manner practically identical to that in which antigenic peptides are bound in the groove of the HLA-DR molecule. It is assumed that the first DR-anchor residue of CLIP is M91 [11]. To make the peptide-DR conforma- 
Cell surface

HLA-DR
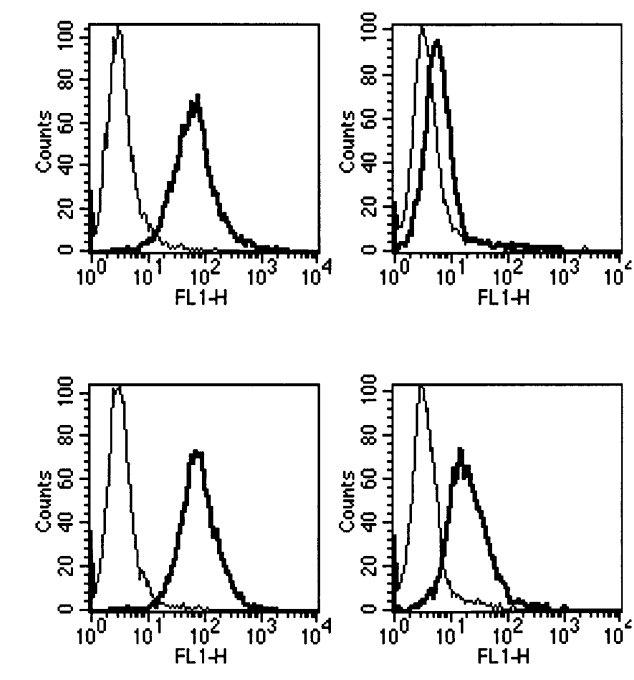

CLIP

LDR4

LDR4-Ii
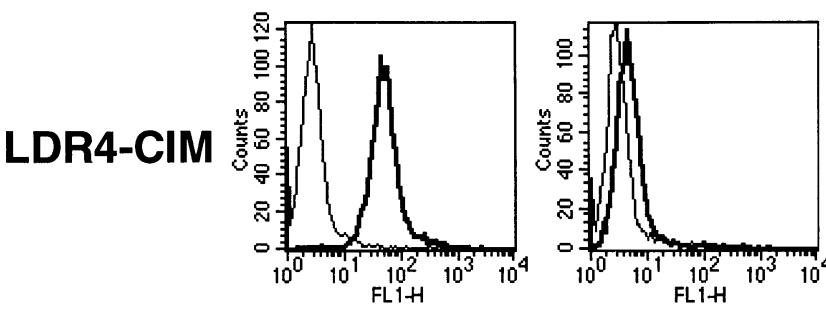

LDR4-FIM
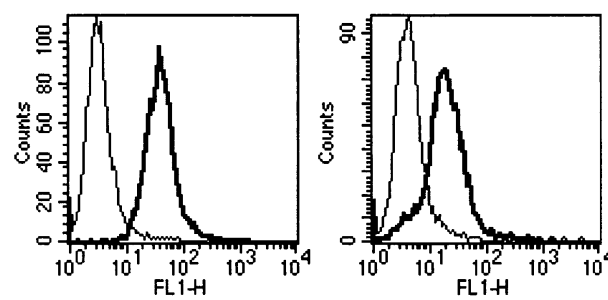

FIGURE 2 Detection of cell surface HLA-DR and CLIP and intracellular invariant chain (Ii). The parental DR4-expressing cell line, LDR4, and cell lines supertransfected respectively with wild-type Ii gene, pCIM and pFIM were analyzed. DR and CLIP were detected by cell surface staining with L243 and CerCLIP.1, respectively. Ii was detected by staining of permeabilized cells with anti-Ii mAb, M-B741. Stained cells were analyzed by flow cytometer and data were presented as fluorescence intensity versus cell number. Isotype matched mAbs were used as negative controls (thin lines). Names of the transfectants are indicated on the side and the molecules detected by $\mathrm{mAbs}$ are indicated at the top of each set of histograms.

tion proper for recognition by YN5-32, we designed the CLIP-substituted Ii construct to locate L57 of M12p55-68 at position M91 in wild-type Ii. The construct of the C-terminal fused Ii vector (pFIM) contains a cathepsin D cleavage site (FLEEKKSG) followed by the

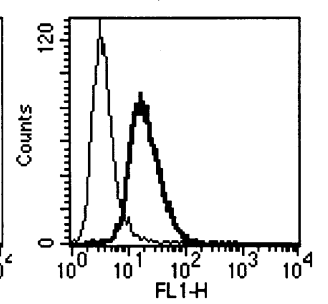

\section{Intracellular}

Invariant chain
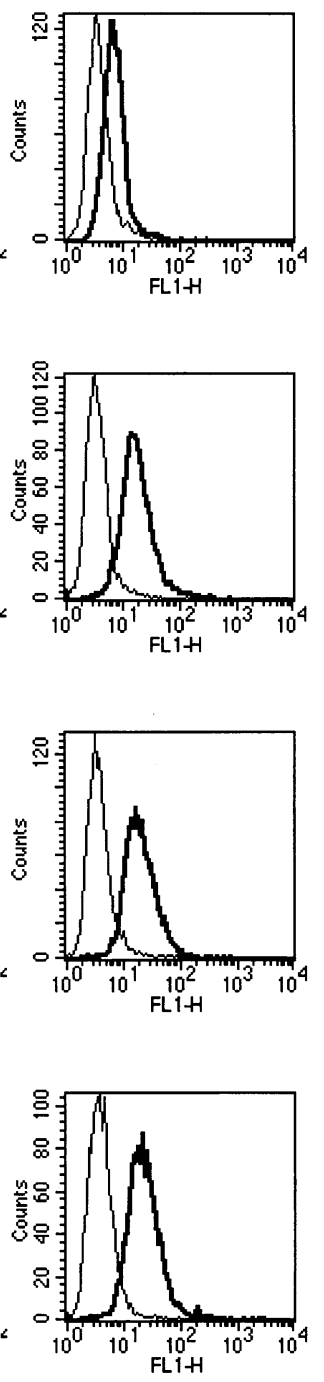

antigenic peptide $\mathrm{M} 12 \mathrm{p} 54-68$ in the $\mathrm{COOH}$-terminus, a strategy reported by Nakano et al. [5].

Expression of Cell Surface HLA-DR and CLIP, and of Intracellular Ii in the Transfected L Cells

Expression of the Ii was detected by intracellular staining of permeabilized cells with the M-B741 mAb. This mAb previously reported to recognize the lumenal domain of human Ii [27] detected the CLIP-substituted Ii as well as wild-type and C-terminal fused $\mathrm{Ii}$, thereby indicating that this $\mathrm{mAb}$ recognizes $\mathrm{I} i$, irrespective of the sequence at the portion of CLIP. As shown in Fig. 2, the four $\mathrm{L}$ cell transfectants expressing comparable levels of HLA-DR and Ii were used for subsequent functional assay. The CerCLIP.1 mAb, specific to CLIP in association with 
FIGURE 3 Association of HLA-DR with Ii. L cell transfectants were labeled for $2 \mathrm{~h}$ with $\left[{ }^{35} \mathrm{~S}\right]$ methionine/cysteine. Cell lysates were immunoprecipitated with anti-DR mAb HU-4. Samples were analyzed by $11 \%$ SDS-PAGE under reduced conditions. The migration positions for Ii p33, the DR $\alpha$ and $\beta$ chains are indicated. Names of the transfectants are given at the top.

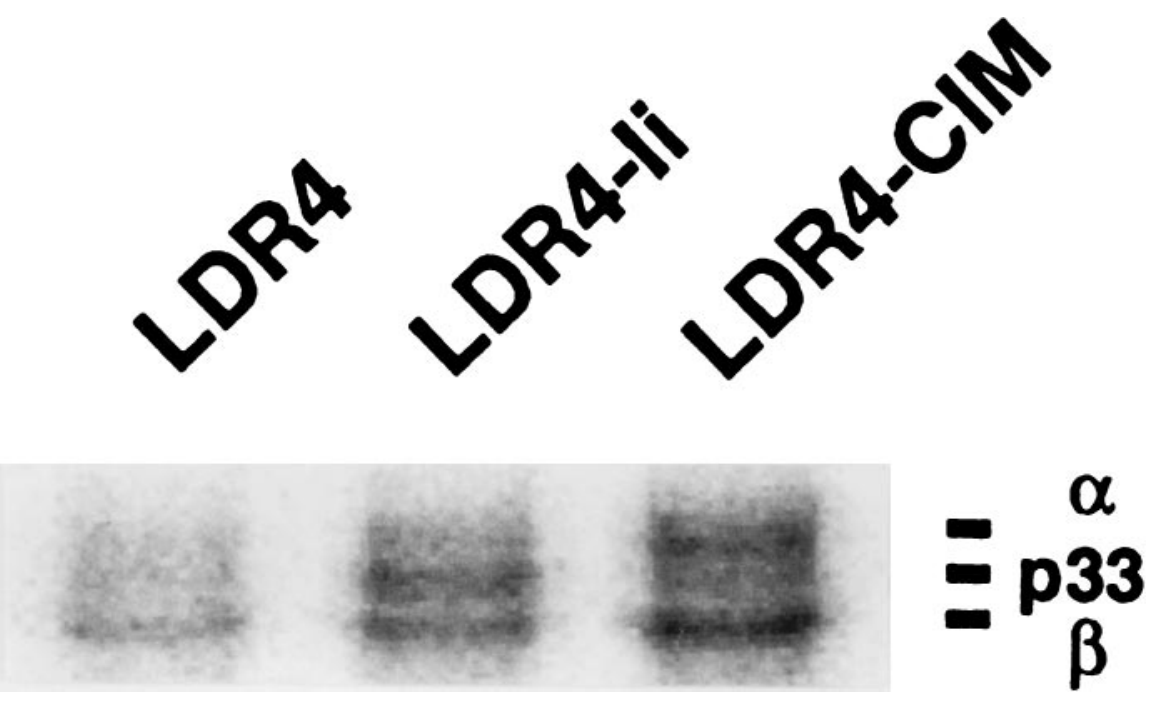

class II molecules, stained wild-type Ii- and C-terminal fused Ii-, but not CLIP-substituted Ii-transfected L cells.

\section{Association of HLA-DR with the Invariant Chain}

It has been reported that Ii-trimer composes a nonameric complex with MHC class II $\alpha$ and $\beta$ chains in the endoplasmic reticulum and functions as a molecular chaperone to target them to endosomes [9]. We asked if the CLIP-substituted Ii was also associated intracellularly with HLA-DR molecules. LDR4, wild-type Ii-LDR4 (LDR4-Ii), and CLIP-substituted Ii-LDR4 (LDR4-CIM) were metabolically labeled with $\left[^{35} \mathrm{~S}\right]$ methionine/cysteine, lysed in mild detergent condition, and precipitated with the anti-HLA-DR mAb (Fig. 3). Both wild-type and CLIP-substituted Ii were coprecipitated with DR, albeit the amount of CLIP-substituted Ii coprecipitated with DR was smaller than that of wild-type Ii, suggesting that CLIP-substituted Ii is not associated with DR as stably as is the wild-type Ii or degraded faster than wild-type Ii. Precipitation with the anti-Ii $\mathrm{mAb}$ also indicated the association of CLIP-substituted Ii with HLA-DR and immunoprecipitates was not detected in LDR4 (data not shown).

\section{Stimulation of the Antigen Specific $\mathrm{CD}^{+}$ T Cell Clone}

To determine if these mutated Ii could load the antigenic peptide on HLA-DR molecules, we tested the proliferative response of the $\mathrm{T}$ cell clone YN5-32. CLIP-substituted Ii-LDR4 (LDR4-CIM) and C-terminal fused Ii-LDR 4 (LDR4-FIM), but not wild-type Ii-LDR4 (LDR4-Ii) and LDR4 stimulated the proliferative response of YN5-32 in the absence of antigenic peptide. T cell proliferation stimulated by LDR4-CIM was approximately twofold over that induced by LDR4-FIM (Fig. 4). To investigate the correlation between expression level of Ii and efficiency of antigen presentation, CLIPsubstituted Ii-LDR 4 with two different expression levels of Ii, high expresser (LDR4-CIM ${ }^{\text {hi }}$ ) and low expresser $\left(\right.$ LDR 4-CIM ${ }^{\mathrm{lo}}$ ), were prepared (Fig. 5A). These two transfectants expressed almost the same amount of DR molecules on the cell surface (data not shown). We tested proliferative responses of YN5-32 induced in the presence of transfectants prepulsed with several different concentrations of M12p54-68 peptide. As shown in Fig. $5 \mathrm{~B}$, at the range of peptide concentrations from 0 to $10^{2}$ $\mu \mathrm{M}$, proliferative response to either $\mathrm{LDR}_{4}-\mathrm{CIM}^{\mathrm{hi}}$ or LDR 4-CIM ${ }^{\mathrm{lo}}$ constantly exceeded that to LDR4-FIM.

FIGURE 4 Stimulation of the antigen specific $T$ cell clone by $\mathrm{L}$ cell transfectants expressing mutated Ii-genes. $\mathrm{T}$ cell clone YN5-32 was cultured in the presence of the indicated L cell transfectants as APCs without the antigenic peptide, and the proliferative response was measured by $\left[{ }^{3} \mathrm{H}\right]$ thymidine incorporation. Results are expressed as means of triplicate determinations \pm standard error. $\left[{ }^{3} \mathrm{H}\right]$ thymidine incorporation into $\mathrm{L}$ cell transfectants alone or $\mathrm{T}$ cell alone did not exceed $100 \mathrm{cpm}$.

$\left[{ }^{3} \mathrm{H}\right]$ thymidine incorporation (cpmx $\times 10^{-3}$ )

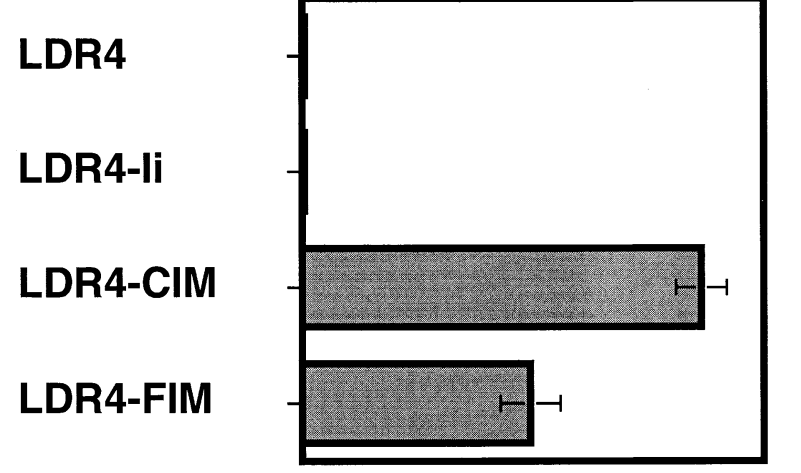


A

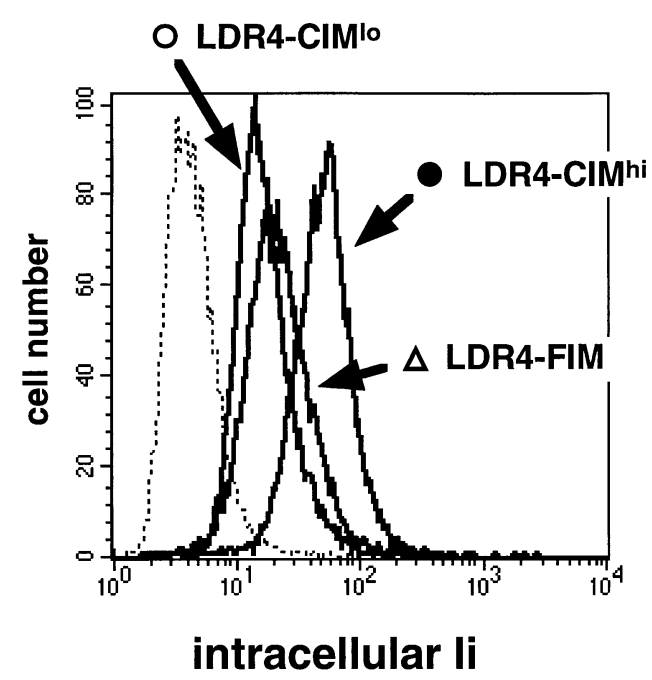

B

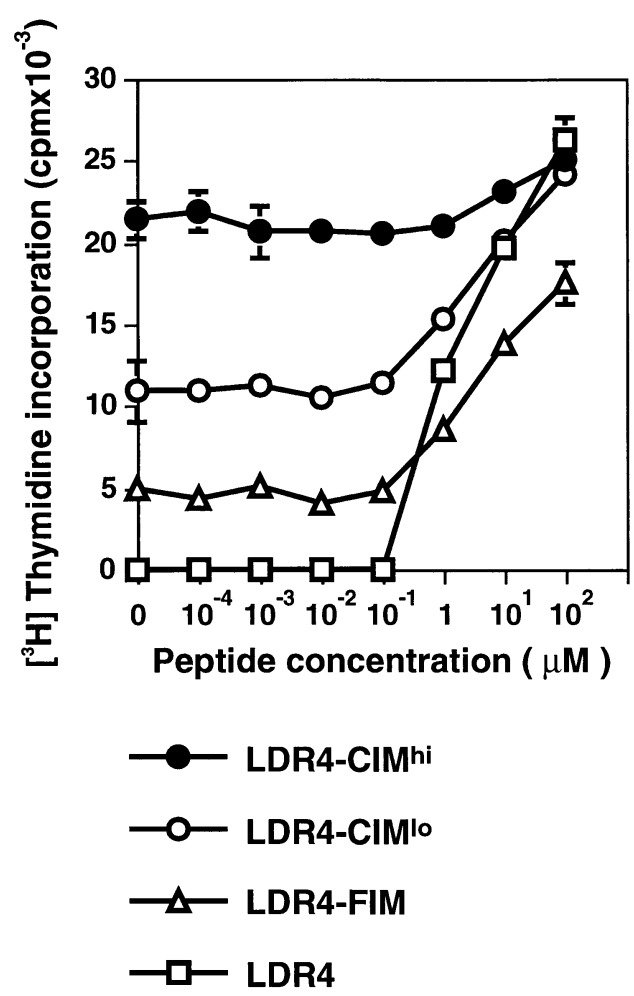

FIGURE 5 Comparison of CLIP-substituted Ii and C-terminal fused Ii for their potential to stimulate a specific $\mathrm{T}$ cell clone. L cell transfectants, including those expressing CLIPsubstituted Ii with different expression levels of intracellular Ii $\left(\mathrm{LDR}_{4}-\mathrm{CIM}^{\mathrm{hi}}\right.$ and LDR4-CIM ${ }^{\mathrm{lo}}$ ), were stained intracellularly with M-B741 (thick lines) or isotype matched mAb as negative controls (thin line) and analyzed by flow cytometry (A). Proliferative response of YN5-32 induced by LDR4-CIM ${ }^{\text {hi }}$, LDR4-CIM ${ }^{\mathrm{lo}}$, C-terminal fused Ii-LDR4 (LDR4-FIM) and LDR 4 in the presence of various concentrations of peptide (B). YN5-32 was cultured with L cell transfectants prepulsed for $16 \mathrm{~h}$ with the indicated concentrations of peptide M12p54-68. Results are expressed as means of triplicate determinations \pm standard errors.
Moreover, proliferative responses to LDR4-CIM ${ }^{\mathrm{hi}}$ reached the approximate plateau level in the absence of antigenic peptide. On the other hand, proliferative responses of YN5-32 to LDR4-CIM ${ }^{\text {lo }}$ and LDR4-FIM increased in a peptide dose-dependent manner in the presence of $10^{-1}-10^{2} \mu \mathrm{M}$ peptide.

\section{DISCUSSION}

We obtained evidence that the CLIP-substituted Ii vector is efficient in loading an antigenic peptide onto MHC class II and stimulating $\mathrm{CD}_{4}^{+} \mathrm{T}$ cells. Almost the same strategy was used by other investigators to present an influenza haemagglutinin-derived peptide to an HLADR1 restricted $\mathrm{CD} 4^{+} \mathrm{T}$ cell clone [6]. Our data show the effectiveness of this strategy for a different antigenic peptide and different allelic product of DR, in comparison with C-terminal fused Ii vector, and in addition, assembly of mutated Ii with DR, suggesting that the inserted antigenic peptide is loaded on the groove of MHC class II in a same way as CLIP, in case of wild-type Ii. The peptide loading process, similar to natural association of CLIP with DR, may provide a higher efficiency when using this vector than that of $\mathrm{C}$-terminal fused Ii vector, as shown in the current study. The CLIP-substituted Ii vector is considered to target the peptide not only to endosomes but also to the groove of MHC-class II. On the other hand, in case of the C-terminal fused Ii vector, the antigenic peptide cleaved out from C-terminus of Ii should locate in the groove of MHC class II and replace CLIP to be presented to $\mathrm{CD} 4^{+} \mathrm{T}$ cells. Because $\mathrm{L}$ cells do not express $\mathrm{H}-2 \mathrm{M}$ molecules [28], the antigenic peptide substituted for CLIP may not be dissociated from DR molecules in LDR4-CIM. On the contrary, replacement of CLIP with an antigenic peptide may not be efficient in LDR4-FIM. This may cause a more acceptable antigen presenting capacity of LDR4-CIM, as compared with LDR4-FIM.

We also found that efficiency of antigen presentation depends on the expression level of CLIP-substituted Ii. The proliferative response of the $\mathrm{T}$ cell clone to LDR4$\mathrm{CIM}^{\mathrm{hi}}$ reached the approximate plateau level in the absence of antigenic peptide, indicating that LDR4-CIM ${ }^{\text {hi }}$ expressed a large number of DR-antigenic peptide complexes on the cell surface sufficient to stimulate full activation of the $\mathrm{T}$ cell clone. On the other hand, the proliferative response of YN5-32 to $\mathrm{LDR}^{-\mathrm{CIM}^{\mathrm{lo}}}$ increased in a peptide dose-dependent manner. This means that density of the DR-antigenic peptide complexes expressed on these two transfectants did not reach a level sufficient to stimulate full activation of $\mathrm{T}$ cells and that empty DR 4 molecules or DR 4 molecules complexed with L cell-derived peptides presented exogenously added antigenic peptides to $\mathrm{T}$ cells. 
The anti-CLIP mAb CerCLIP.1 recognizes CLIP in association with HLA class II molecules [23]. Staining with CerCLIP.1 showed that LDR4-Ii and LDR4-FIM expressed class II $\alpha \beta$-CLIP complex similar to T2, a human cell line deleted in HLA-linked genes including HLA-DM [29], but not LDR4-CIM. This observation supports an notion that CerCLIP. 1 recognizes CLIP residues 89-102 in the context of HLA-DR. Alternatively it is possible that the $\mathrm{mAb}$ cannot recognize an epitope on CLIP 81-89 in LDR4-CIM because of an alteration in conformation by substitution at residues 89-102.

Modulation of immune responses by stimulating or antagonizing specific $\mathrm{T}$ cell populations in vivo may be one avenue to use when designing treatment of subjects with allergic or autoimmune diseases or even for anticancer immunotherapy. It would be essential to identify peptides which can stimulate pathogenic $\mathrm{T}$ cells. We are using pCI, our CLIP-substituted Ii vector, to establish an expression library system for use in searching epitopes for specific $\mathrm{CD}^{+}{ }^{+} \mathrm{T}$ cells. A different type of vector to load an antigenic peptide onto MHC class II has been reported by Ignatowicz et al. [30], in which the peptide was covalently linked to the $\mathrm{N}$-terminus of the $\beta$ chain of MHC class II. pCI seems to be more useful than the $\beta$ chain-linked vector in that, once established, the pCI library can be applied to various allelic products and classes of MHC class II molecules. Although the epitope identified by this strategy is not necessarily the natural ligand of the $\mathrm{T}$ cells, it is possible that the epitope works as well as or even more efficiently than natural epitopes.

$\mathrm{pCI}$ may also prove useful to present specific epitope to $\mathrm{CD}^{+}{ }^{+} \mathrm{T}$ cells in vivo, as a DNA vaccine. Infusion of synthetic peptides or peptide-pulsed antigen presenting cells may also be used for that purpose. However, DNA transfer of a pCI encoding specific peptide is thought to be more efficient and longer lasting, because the epitope is continuously produced and is loaded onto MHC class II in vivo.

\section{ACKNOWLEDGMENTS}

This present work was supported by Grants-in Aid 08282104, 08557027, and 09470097 from the Ministry of Education, Science, Sports and Culture, Japan; a Research Grant for Intractable Diseases from the Ministry of Health and Welfare, Japan; Japan Rheumatism Foundation, Mochida Memorial Foundation, and The Naito Foundation. We thank Dr. P. Creswell (Yale university) for anti-CLIP mAb CerCLIP.1, Dr. Y. Nakabeppu (Kyushu university) for pYN3214-lacZ, and M. Ohara for helpful comments.

\section{ADDENDUM}

After this work was completed a similar approach was presented by Malcherek $G$ et al., Eur J Immunol 28: 1524, 1998.

\section{REFERENCES}

1. York IA, Rock KL: Antigen processing and presentation by the class I major histocompatibility complex. Ann Rev Immunol 14:369, 1996.

2. Parra-Lopez CA, Lindner R, Vidavsky I, Gross M, Unanue ER: Presentation on class II MHC molecules of endogenous lysozyme targeted to the endocytic pathway. J Immunol 158:2670, 1997.

3. Wu TC, Guarnieri FG, Staveley OCKF, Viscidi RP, Levitsky HI, Hedrick L, Cho KR, August JT, Pardoll DM: Engineering an intracellular pathway for major histocompatibility complex class II presentation of antigens. Proc Natl Acad Sci USA 92:11671, 1995.

4. Sanderson S, Frauwirth K, Shastri N: Expression of endogenous peptide-major histocompatibility complex class II complexes derived from invariant chain-antigen fusion proteins. Proc Natl Acad Sci USA 92:7217, 1995.

5. Nakano N, Rooke R, Benoist C, Mathis D: Positive selection of $\mathrm{T}$ cells induced by viral delivery of neopeptides to the thymus. Science 275:678, 1997.

6. van Bergen J, Schoenberger SP, Verreck F, Amons R, Offringa R, Koning F: Efficient loading of HLA-DR with a T helper epitope by genetic exchange of CLIP. Proc Natl Acad Sci USA 94:7499, 1997.

7. Strubin M, Long EO, Mach B: Two forms of the Ia antigen-associated invariant chain result from alternative initiations at two in-phase AUGs. Cell 47:619, 1986.

8. O'Sullivan DM, Noonan D, Quaranta V: Four Ia invariant chain forms derive from a single gene by alternate splicing and alternate initiation of transcription/translation. J Exp Med 166:444, 1987.

9. Cresswell P: Assembly, transport, and function of MHC class II molecules. Ann Rev Immunol 12:259, 1994.

10. Roche PA, Cresswell P: Proteolysis of the class II-associated invariant chain generates a peptide binding site in intracellular HLA-DR molecules. Proc Natl Acad Sci USA 88:3150, 1991.

11. Ghosh P, Amaya M, Mellins E, Wiley DC: The structure of an intermediate in class II MHC maturation: CLIP bound to HLA-DR3. Nature 378:457, 1995.

12. Roche PA, Marks MS, Cresswell P: Formation of a ninesubunit complex by HLA class II glycoproteins and the invariant chain. Nature 354:392, 1991.

13. Bakke O, Dobberstein B: MHC class II-associated invariant chain contains a sorting signal for endosomal compartments. Cell 63:707, 1990.

14. Pond L, Kuhn LA, Teyton L, Schutze MP, Tainer JA, Jackson MR, Peterson PA: A role for acidic residues in di-leucine motif-based targeting to the endocytic pathway. J Biol Chem 270:19989, 1995.

15. Peters PJ, Neefjes JJ, Oorschot V, Ploegh HL, Geuze HJ: Segregation of MHC class II molecules from MHC class I molecules in the Golgi complex for transport to lysosomal compartments. Nature 349:669, 1991. 
16. Tulp A, Verwoerd D, Dobberstein B, Ploegh HL, Pieters $\mathrm{J}$ : Isolation and characterization of the intracellular MHC class II compartment. Nature 369:120, 1994.

17. Amigorena S, Drake JR, Webster P, Mellman I: Transient accumulation of new class II MHC molecules in a novel endocytic compartment in B lymphocytes. Nature 369: 113, 1994.

18. Denzin LK, Cresswell P: HLA-DM induces CLIP dissociation from MHC class II alpha beta dimers and facilitates peptide loading. Cell 82:155, 1995.

19. Sloan VS, Cameron P, Porter G, Gammon M, Amaya M, Mellins E, Zaller DM: Mediation by HLA-DM of dissociation of peptides from HLA-DR. Nature 375:802, 1995.

20. Chen YZ, Matsushita S, Nishimura Y: Response of a human $\mathrm{T}$ cell clone to a large panel of altered peptide ligands carrying single residue substitutions in an antigenic peptide: characterization and frequencies of TCR agonism and TCR antagonism with or without partial activation. J Immunol 157:3783, 1996.

21. Gregersen PK, Goyert SM, Song QL, Silver J: Microheterogeneity of HLA-DR4 haplotypes: DNA sequence analysis of LD“KT2" and LD“TAS" haplotypes. Hum Immunol 19:287, 1987.

22. Inoko H, Bodmer JG, Heyes JM, Drover S, Trowsdale J, Marshall WH: Joint report on the transfectant/monoclonal antibody component. In Tsuji K, Aizawa M, Sasazuki T (eds). HLA 1991 vol 1, Oxford, Oxford University Press, 1992.

23. Denzin LK, Robbins NF, Carboy-Newcomb C, Cresswell P: Assembly and intracellular transport of HLA-DM and correction of the class II antigen-processing defect in T2 cells. Immunity 1:595, 1994.
24. Takebe Y, Seiki M, Fujisawa J, Hoy P, Yokota K, Arai K, Yoshida M, Arai N: SR alpha promoter: an efficient and versatile mammalian cDNA expression system composed of the simian virus 40 early promoter and the R-U5 segment of human T-cell leukemia virus type 1 long terminal repeat. Mol Cel Biol 8:466, 1988.

25. Ikagawa S, Matsushita S, Chen YZ, Ishikawa T, Nishimura Y: Single amino acid substitutions on a Japanese cedar pollen allergen (Cry j 1)-derived peptide induced alterations in human $\mathrm{T}$ cell responses and $\mathrm{T}$ cell receptor antagonism. J Allergy Clin Immunol 97:53, 1996.

26. Kamikawaji N, Fujisawa K, Yoshizumi H, Fukunaga M, Yasunami M, Kimura A, Nishimura Y, Sasazuki T: HLADQ-restricted $\mathrm{CD}_{4}^{+} \mathrm{T}$ cells specific to streptococcal antigen present in low but not in high responders. J Immunol 146:2560, 1991.

27. Möller P, Mordenhauer G: B-cell antigens: CD74 workshop panel report: In Kishimoto T, Kikutani $\mathrm{H}$, von dem Borne AEGK, Goyert SM, Mason DY, Miyasaka M, Moretta L, Okumura K, Shaw S, Springer TA, Sugamura K, Zola H (eds): Leucocyte typing VI. p. 166. New York, Garland Publishing Inc, 1997.

28. Katz JF, Stebbins C, Appella E, Sant AJ: Invariant chain and DM edit self-peptide presentation by major histocompatibility complex (MHC) class II molecules. J Exp Med 184:1747, 1996.

29. Riberdy JM, Newcomb JR, Surman MJ, Barbosa JA, Cresswell P: HLA-DR molecules from an antigen-processing mutant cell line are associated with invariant chain peptides. Nature 360:474, 1992.

30. Ignatowicz L, Winslow G, Bill J, Kappler J, Marrack P: Cell surface expression of class II MHC proteins bound by a single peptide. J Immunol 154:3852, 1995. 